\title{
Effects of nerve growth factor and basic fibroblast growth factor dual gene modification on rat bone marrow mesenchymal stem cell differentiation into neuron-like cells in vitro
}

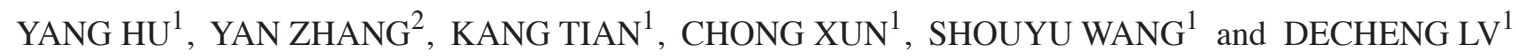 \\ ${ }^{1}$ Department of Orthopedics, The First Hospital Affiliated to Dalian Medical University, Dalian, Liaoning 116011; \\ ${ }^{2}$ Institute of Cancer Stem Cells, Cancer Center, Dalian Medical University, Dalian, Liaoning 116044, P.R. China
}

Received April 28, 2014; Accepted August 26, 2015

DOI: $10.3892 / \mathrm{mmr} .2015 .4553$

\begin{abstract}
Recent studies regarding regenerative medicine have focused on bone marrow mesenchymal stem cells (BMSCs), which have the potential to undergo neural differentiation, and may be transfected with specific genes. BMSCs can differentiate into neuron-like cells in certain neurotropic circumstances in vitro. Basic fibroblast growth factor (bFGF) and nerve growth factor (NGF) are often used to induce neural differentiation in BMSCs in vitro. However, previous studies regarding their combined actions are insufficient. The present study is the first, to the best of our knowledge, to thoroughly assess the enhancement of neural differentiation of BMSCs following transfection with bFGF and NGF. Sprague-Dawley (SD) rat BMSCs were separated through whole bone marrow adherence, and were then passaged to the third generation. The cells were subsequently divided into five groups: The control group, which consisted of untransfected BMSCs; the plv-blank-transfected BMSCs group; the plv-bFGF-transfected BMSCs group; the plv-NGF-transfected BMSCs group; and the plv-NGF-bFGF co-transfected BMSCs group. Cell neural differentiation was characterized in terms of stem cell molecular expression, and the neuronal morphology and expression of neural-like molecules was detected in each of the groups. A total of $72 \mathrm{~h}$ post-transfection, the expression levels of neuron-specific enolase, glial fibrillary acidic protein, and nestin protein, were higher in the co-transfected group, as compared with the other groups, the expression levels of $\beta$-tubulin III were also increased in the co-transfected cells, thus suggesting the maturation of differentiated neuron-like
\end{abstract}

Correspondence to: Dr Shouyu Wang or Professor Decheng Lv, Department of Orthopedics, The First Hospital Affiliated to Dalian Medical University, 222 Zhangshan Road, Dalian, Liaoning 116011, P.R. China

E-mail: 1vdechengprofessor@163.com

E-mail: wangshouyu666.student@sina.com

Key words: nerve growth factor, basic fibroblast factor, bone marrow mesenchymal stem cells, neuron-llike cell cells. Furthermore, higher neuronal proliferation was observed in the co-transfected group, as compared with the other groups at passages 2, 4, 6 and 8. Western blotting demonstrated that the transfected groups exhibited a simultaneous increase in phosphorylation of the AKT and extracellular signal-regulated kinases (ERK) signaling pathway. These results suggested that manipulation of the ERK and AKT signaling pathway may be associated with the differentiation of transfected BMSCs.

\section{Introduction}

A promising future for the use of bone marrow mesenchymal stem cells (BMSCs) in regenerative medicine is anticipated, due to their ability to self-replicate and differentiate into various functional cells. Stem cells are the most widely used seed cells in the field of neural regeneration and bone tissue engineering, due to their easy availability, reduced side effects, biological scaffold adhesion abilities, and promising performances in vitro, as well as their strong proliferative capacity in culture and passaging (1-6). Therefore, stem cells are currently being widely investigated and applied in the clinic (1). BMSCs possess powerful differentiation potential and immunological regulatory properties, and therefore have potential for use in the field of cell injury. Numerous studies have demonstrated that BMSCs are able to differentiate into neuron-like cells in vitro via genetic manipulation, where various factors and chemical agents are adopted to induce BMSC differentiation into neuron-like or Schwann cells (1-3,7). However, due to ethical considerations and the various methods used to induce adult stem cell neural differentiation, how to produce and identify the source of neuron-like cells for clinical cell therapy is currently a significant problem.

BMSCs transplanted in adult Sprague-Dawley (SD) rat may respond to microenvironmental cues and differentiate into neuron-like cells. In addition, BMSCs have been shown to migrate towards the source of lesions in the brain (4), which may enhance the repairing capacity of injured tissue in animal models. A previous study demonstrated that BMSC transplantation into the central nervous system was able to impede Alzheimer's disease-like pathology and upregulate \$Np73 expression in the hippocampus of APP/PS1 transgenic mice (5). In addition, Mohammadi et al $(2,8)$ used 
undifferentiated bone marrow stromal cells to induce sciatic nerve regeneration in rats. Administration of BMSCs via the central nervous system and peripheral system is considered to be safe in human subjects (8-10). BMSCs may become a clinical choice for cell therapy of the central nervous and peripheral systems, since BMSCS have the advantage of reduced ethical regulation and do not often induce tissue rejection. The rapid development of nerve engineering technology has enabled many investigators to examine the use of natural and artificial biomaterials. Constructed grafts may be used to connect and repair in neurological regeneration (9-11); however, the new nerve must possess biocompatibility. Conversely, stem cells have the ability to secrete neurotropic factors to repair injured neurons. BMSCs are not prone to ethical and tissue rejection-related concerns; however, further studies on the use of human BMSCs are required.

Basic fibroblast growth factor (bFGF) and nerve growth factor (NGF) are powerful mitogens that promote the nutrition of neural stem cells and precursor cells present in the mature nervous system. Through the expression of nerve-related proteins, bFGF promotes cell proliferation and mitosis, and enhances neuronal axon regeneration and spinal cord injury repair (12). NGF is a homodimeric peptide. By supporting the survival and growth of neural cells in the nervous system, it is able to regulate cell growth and promote neural differentiation. Furthermore, NGF exhibits nerve injury healing ability in clinical therapy (13). BMSCs may be stably transfected in order to overexpress exogenous genes. According to a previous experiment, transfected BMSCs are capable of differentiating into endodermal and ectodermal cells (14). It has also been reported that BMSCs transplanted into neonatal mice brain may differentiate into neurons and glial cells (15-19). However, the differentiation rate of BMSCs into neuron-like cells is much lower, as compared with other types of differentiated cells; therefore, the present study aimed to increase the efficiency of BMSC neural differentiation in vitro (14-17).

There are numerous chemical reagents and cytokines widely used to induce the differentiation of neural BMSCs in vitro, such as dimethyl sulfoxide, $\beta$-mercaptoethanol, vitamin $\mathrm{C}$, insulin, valproic acid, forsklin, hydrocortisone and NT-3 retinoic acid (18). Epidermal growth factor (EGF) and bFGF are powerful mitogens and cell fate drivers for neural precursor cells $(19,20)$, whereas bFGF may maintain a neurogenic microenvironment in vivo (21).

NGF is a type of neurotrophin, which exerts an anti-apoptotic function in premature neurons (13). Based on effective biological activation, NGF is associated with the neural differentiation and migration of neural cells. In addition, NGF can protect axons and myelin from inflammatory damage in order to modulate the immune system, as well as protect and enhance excitotoxicity during inflammatory activation. It has been demonstrated that NGF can induce BMSC differentiation into neural cells, via generating neuropeptide signals and receptors (6). These findings suggest that NGF is essential for BMSC neural differentiation, which may be beneficial for the treatment of injured nerves. The present study used NGF and bFGF recombinant lentiviral vectors to transfect BMSCs in vitro, and the neural differentiation efficiency of the BMSCs was investigated. The aim of the present study was to observe the differentiation of the co-transfected BMSCs into neuron-like cells in vitro.

\section{Materials and methods}

Cell isolation and harvest. BMSCs were isolated and harvested according to a previous method (22-24). Briefly, male and female SD rats (age, 2-3 weeks, weighing 80-100 g) were sacrificed. Their bone marrows were harvested by flushing the marrow cavities with $5 \mathrm{ml}$ Dulbecco's modified Eagle's medium (DMEM)/F12 supplemented with $10 \%$ fetal bovine serum (FBS; Hyclone, GE Healthcare Life Sciences, Logan, UT, USA) and $1 \%$ penicillin/streptomycin. The marrow cavities were then rinsed two to three times until white. The sample was transferred to $15 \mathrm{ml}$ sterile centrifuge tubes, which were centrifuged at $300 \mathrm{x}$ g for $5 \mathrm{~min}$. The precipitate was resuspended in fresh DMEM/F12 (Hyclone) medium containing 10\% FBS (Hyclone), penicillin (100 U/ml (Hyclone), and streptomycin (100 g/l; Hyclone). Subsequently, the cells were seeded at a density of $1 \times 10^{4}$ in $10 \mathrm{~cm}^{2}$ flasks (Falcon Plastics, Oxnard CA, USA) and cultured at $37^{\circ} \mathrm{C}$ in a humidified atmosphere containing $5 \% \mathrm{CO}_{2}$. After 3 days, the medium was replaced and non-adherent cells were removed. Spindle-shaped adherent MSCs were expanded and purified with 3-5 passages after initial plating. The medium was then replaced and the cells were observed under an Olympus 81 inverted phase contrast microscope (Olympus Corporation, Tokyo, Japan) every 3 days. After 12 days, spindle-shaped adherent BMSCs were harvested with $0.25 \%$ trypsin (Hyclone, GE Healthcare Life Sciences) and subcultured once they reached $80-90 \%$ confluence. The third or fourth generation BMSCs were digested using $0.25 \%$ EDTA-trypsin and seeded in six well plates containing poly-L-lysine coverslips at a density of $1 \times 10^{6}$ cells/well. This study was approved by the ethics committee of the Institutional Animal Care and Use Committee of Dalian Medical University (Dalian, China).

Flow cytometric analysis. For flow cytometry of the isolated BMSCs, BMSC cell markers, including CD29, CD34, CD44, CD45, CD71, CD90 and CD106 (BD Biosciences, Franklin Lakes, NJ, USA) were considered positive, whereas CD45 was considered negative. The following mouse monoclonal $\mathrm{CD}$ antibodies were conjugated with fluorescein isothiocyanate (FITC; BD Biosciences): Anti-CD29 (eBioscience, San Diego, CA, USA; cat. no. 12-0291); -CD34 (eBioscience; cat. no. 12-0341); -CD44 (eBioscience; cat. no. 12-0441) -CD45 (eBioscience; cat. no. 11-0451), -CD71 (eBioscience; cat. no. 11-0711), -CD90 (eBioscience; cat. no. 11-0900); and -CD106 (eBioscience; cat. no. 11-4321). The surfaces of the fourth passage BMSCs were stained; BMSC suspensions $\left(1 \times 10^{5}\right)$ were mixed with $10 \mu \mathrm{l}$ FITC, and incubated in a dark room at $4^{\circ} \mathrm{C}$ for $30 \mathrm{~min}$. The cells were then analyzed using a flow cytometer (BD FACSDiva Software version 6.1.3; BD ARIA II and Diva software; BD Biosciences).

Plasmid construction and transduction. Lentiviral-plasmids encoding human NGF (NM_002506.2) and human bFGF2 (NM_002006.4) (Cyagen Biotechnology, Co., Ltd., Guangzhou, China) were generated by PCR amplification and subcloned into the pLV.Des3d.P/puro expression 
vector (Invitrogen Life Technologies, Carlsbad, CA, USA). The plasmids pLV. ExSi.P/Puro-CMV-NGF-T2A, pLV.ExSi.P/Puro-CMV-FGF2-P2A, pLV.ExSi.P/PuroCMV-NGF-T2A-FGF2-P2A and pLV.ExSi.P/Puro-CMV were constructed as previously described (25). Expression plasmids were transfected into the 293T cells (Cyagen Biosciences, Sunnyvale, CA,USA) using Lipofectamine ${ }^{\circledR} 2000$ (Invitrogen Life Technologies), according to the manufacturer's instructions. Briefly, overexpression of the specific delivered gene was generated using a lentiviral system (Sigma-Aldrich, St. Louis, MO, USA), according to the manufacturer's instructions. Briefly, to generate the specific NGF- and bFGF-containing lentiviruses, 1,077 x10 $293 \mathrm{~T}$ cells were co-transfected with pC9MV-VSV-G $(15 \mu \mathrm{g})$, PRSV-Rev $(15 \mu \mathrm{g})$ and pMDLg/pRRE $(15 \mu \mathrm{g})$. Transduction of 293T cells with compatible packaging plasmids and $15 \mu \mathrm{g}$ plv plasmids was conducted in $10-\mathrm{cm}^{2}$ flasks for $24 \mathrm{~h}$ using Lipofectamine $^{\circledR} 2000$ transduction reagent $(100 \mu \mathrm{l})$ diluted in OPTI-MEM (1.5 ml; Thermo Fisher Scientific, Inc., Waltham, MA, USA). Following $16 \mathrm{~h}$ of transduction, the media was replaced with OPTI-MEM containing $10 \mathrm{mM}$ sodium butyrate. At $24 \mathrm{~h}$ post-transduction, the media was replaced with viral harvesting medium. Subsequently, the viruses were harvested and the media was replaced with viral harvesting medium.

Harvesting of viruses and titration of lentiviral vectors. BMSCs $\left(3 \times 10^{5}\right.$ cells) were seeded in a $6 \mathrm{~cm}$ plate and incubated at $37^{\circ} \mathrm{C}$ for $24-48 \mathrm{~h}\left(5 \% \mathrm{CO}_{2}\right)$ until the cells reached $30-50 \%$ confluence. The medium was then replaced with serum-free medium, and transduction was performed $2 \mathrm{~h}$ later. Briefly, the medium was removed and $1 \mathrm{ml}$ virus-containing supernatant and $1 \mu \mathrm{l}$ polybrene $(8 \mathrm{mg} / \mathrm{ml}$; Santa Cruz Biotechnology Inc., Dallas, TX, USA) was added with $3 \mathrm{ml}$ complete medium, and the cells were incubated for $2 \mathrm{~h}$. The cells were then allowed to grow for 2 days.

Western blotting. In order to detect the protein expression levels of the two genes western blotting was performed. The transfected BMSCs differentiated at various passages were lysed in radioimmunoprecipitation buffer for $72 \mathrm{~h}$. The proteins were then extracted from SDS-PAGE and transferred to polyvinylidene difluoride membranes, which were incubated with $5 \%$ skim-milk in phosphate-buffered saline (PBS) for $1 \mathrm{~h}$ at $37^{\circ} \mathrm{C}$ to reduce non-specific binding. The membranes were washed with PBS containing $0.1 \%$ Tween-20, and were incubated with antibodies targeting NGF (1:500; cat. no. sc-33602; Santa Cruz Biotechnology, Inc.), bFGF (1:1,000; cat. no. sc-79; Santa Cruz Biotechnology, Inc.), $\beta$-tubulin III (1:500; cat. no. MFCD01323870; Sigma-Aldrich), GAPDH (1:5,000; cat. no. sc-25778; Santa Cruz Biotechnology, Inc.), Akt (1:1,000; cat. no. 9272; Cell Signaling Technology, Inc., Danvers, MA, USA), phosphorylated (p)-Akt (1:1,000; cat. no. 9271; Cell Signaling Technology, Inc.), extracellular-signal regulated kinases (ERK; 1:1,000; cat. no. 9102; Cell Signaling Technology, Inc.), and p-ERK (1:1,000; cat. no. 9101; Cell Signaling Technology) overnight at $4^{\circ} \mathrm{C}$. The membranes were then incubated with the corresponding secondary antibodies. The blots were developed using enhanced chemiluminescence western blotting reagents, and were visualized using the Bio-Rad Image Lab system (Bio-Rad Laboratories, Inc., Hercules, CA, USA).

BMSC groups. The BMSCs were divided into five groups: The control group, which consisted of untransfected BMSCs; the plv-blank-transfected BMSCs group; the plv-bFGF-transfected BMSCs group; the plv-NGF-transfected BMSCs group; and the plv-NGF-bFGF co-transfected BMSCs group. All of the groups were cultured in DMEM/F12 (Thermo Fisher Scientific, Inc.) supplemented with $20 \%$ FBS, $1 \%$ penicillin and streptomycin $(100 \mathrm{U} / \mathrm{ml})$. When the cells had proliferated and reached $80 \%$ confluency, the differentiation time was between 1 and 7 days until the next passage. Subsequently, each group was fixed for immunocytochemistry.

Detection of neuron-like cells induced from transfected BMSCs using immunofluorescence. Transfected fourth passage BMSCs were digested with $0.25 \%$ EDTA-trypsin. The cells $\left(1 \times 10^{6}\right)$ were then seeded in 6 -well plates containing poly-D-lysine-coated cover-glass, until all of the cells were induced to neuronally differentiate. After $72 \mathrm{~h}$, the cells were washed with PBS. The plates were then treated with $1 \mathrm{ml} 4 \%$ paraformaldehyde for $15 \mathrm{~min}$, washed with PBS and fixed with $3.7 \%$ formaldehyde in PBS for $25 \mathrm{~min}$. Subsequently, the plates were rinsed with PBS, and permeated with $0.1 \%$ Triton X-100 (Solarbio Beijing China) in $1 \mathrm{ml}$ PBS for $20 \mathrm{~min}$. The plates were then washed with PBS for $1 \mathrm{~h}$, blocked with $1 \%$ bovine serum albumin in PBS for $1 \mathrm{~h}$, and rinsed three times in PBS, each for $10 \mathrm{~min}$. The plates were incubated overnight with primary anti-nestin (1:200; Santa Cruz Biotechnology, Inc.), anti-glial fibrillary acidic protein (GFAP; 1:200; Wuhan Boster Biological Technology, Ltd., Wuhan, China), anti-neuron specific enolase (NSE; 1:200; Wuhan Boster Biological Technology, Ltd.), and anti- $\beta$-tubulin III (1:500; Sigma-Aldrich) in blocking solution for $2 \mathrm{~h}$. The slides were washed with blocking solution three times for $10 \mathrm{~min}$ each time, and the plates were incubated for $2 \mathrm{~h}$ with secondary antibodies conjugated to Alexa Fluor $488(2 \mu \mathrm{g} / \mathrm{ml}$, Invitrogen Life Technologies) in blocking solution. Subsequently, the plates were washed with blocking solution three times for $10 \mathrm{~min}$ each, and were incubated with $1 \mu \mathrm{g} / \mathrm{ml}$ DAPI (Invitrogen Life Technologies) in blocking solution for $2 \mathrm{~min}$. The membranes were washed a further three times with PBS and dried, and the cells were observed under a fluorescence microscope (DP73; Olympus Corporation). $\beta$-tubulin III was observed using a confocal laser scanning microscope (TCS SP5 II; Leica Microsystems GmbH, Wetzlar, Germany).

Statistical analysis. Data are presented as the mean \pm standard deviation. Standard analyses were carried out using an independent-samples t-test. Experimental data were analyzed by SPSS version 19.0 (IBM, Armonk, NY, USA). P<0.05 was considered to indicate a statistically significant difference.

\section{Results}

Identification of BMSCs isolated from SD rats. The morphology of BMSCs isolated from SD rats was altered after the initial passage, as observed under an inverted microscope 


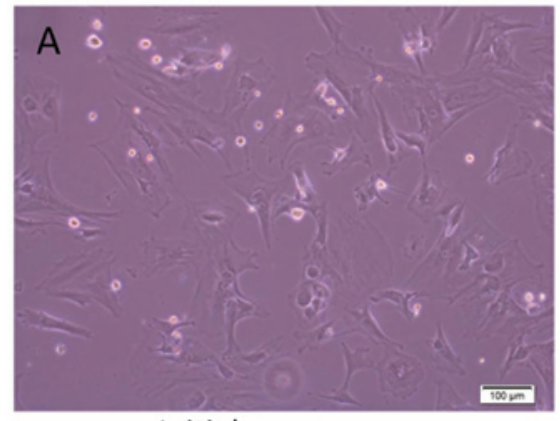

Initial passage

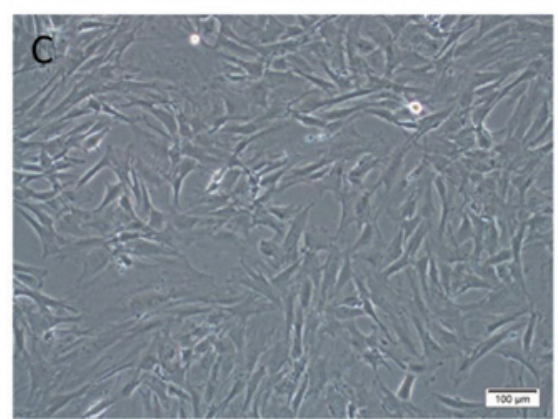

Third passage

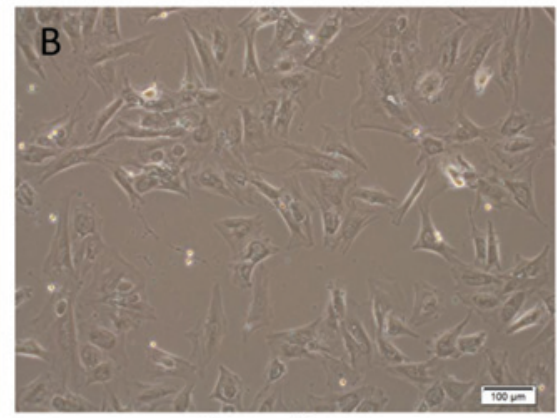

Second passage

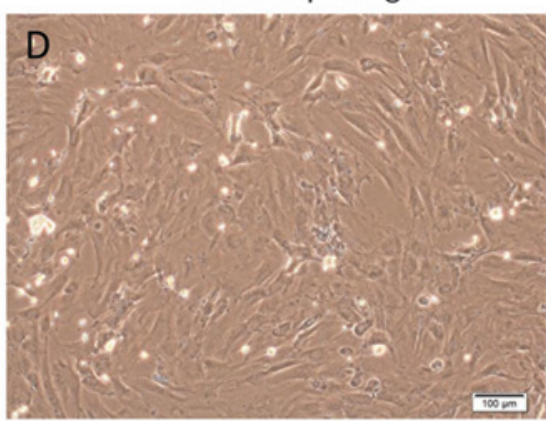

Fourth passage

$\mathbf{E}$

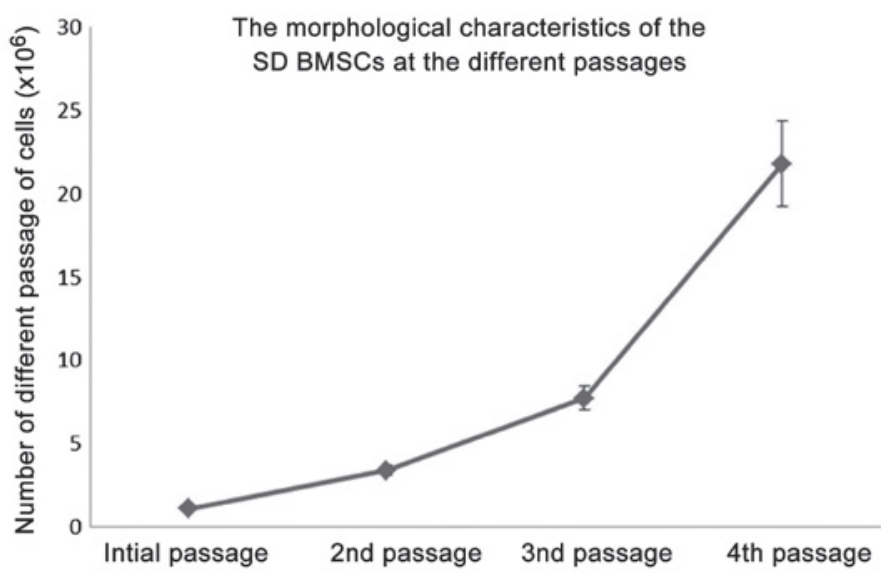

Figure 1. Morphological characteristics of the bone marrow mesenchymal stem cells (BMSCs) isolated from the Sprague-Dawley (SD) rats at various passages. (A) Representative BMSCs of the initial passage with small-round, flat and fibroblast-like morphology. (B) Representative cells with small rounded morphology, large flattened morphology, and fibroblast-like morphology are observed in the 3rd and 4th passages, respectively. (C) BMSC morphology had gradually shifted to a bipolar, spindle-like shape at the third passage. (D) Fourth passage BMSCs exhibited a uniform fibroblast-like, long-spindle shape, and continuously proliferated. Phase contrast microscopy; scale bars represent $100 \mu \mathrm{m}$. (E) The number of cells at each passage was counted from BMSCs isolated from six SD rats.

(Fig 1). As shown in Fig 1, the primary BMSCs were oval-like and spindle-shaped, and some adherent fibroblast cells were observed. Synaptic-like morphological changes occurred in the cells, which gradually fused with each other following the replacement of culture medium over the following days. As compared with the initial cells, the second passage cells appeared larger, and as they proliferated they extended from a triangle to polygon shape. During the second passage, the rate of proliferation was increased and the cells were shown to contain 1-2 visible round and large nucleoli. While the cells are found to be integrated minimally with the surrounding for shape and distribution, the proliferation rate was significantly increased, as compared with in the original cells. The BMSCs were especially easy to subculture during the third passage. At the fourth passage increased cell proliferation, polygonal cell morphology and long fusiform growth were observed.
Identification of BMSCs. In previous studies, numerous antigenic markers have been reported to be expressed in BMSCs rather than a single specific antigenic marker (24). According to previous studies (24), CD29 and CD44 are regarded as the common positive antigen markers that may be used to identify mouse BMSCs. In the present study, even more CD markers were analyzed, in order to identify fourth passage BMSCs. The results of the flow cytometric analysis on fourth passage BMSCs using CD29, CD44, CD90, CD105, CD34, CD45, and CD106 monoclonal cell surface antibodies are shown in Fig. 2A. Fig. 2B presents the percentage of positively-stained cells for each antigen marker: CD29 (99.25 $\pm 0.82 \%)$, CD34 $(1.00 \pm 0.20 \%)$, CD44 (83.73 $\pm 4.95 \%)$, CD45 (0.98 $\pm 0.19 \%)$, CD71 (89.86 $\pm 2.39 \%)$, CD90 $(99.21 \pm 0.79 \%)$ and CD106 $(86.42 \pm 5.67 \%)$. These results suggested that the extracted BMSCs were high-purity, easily harvested, and stable. 


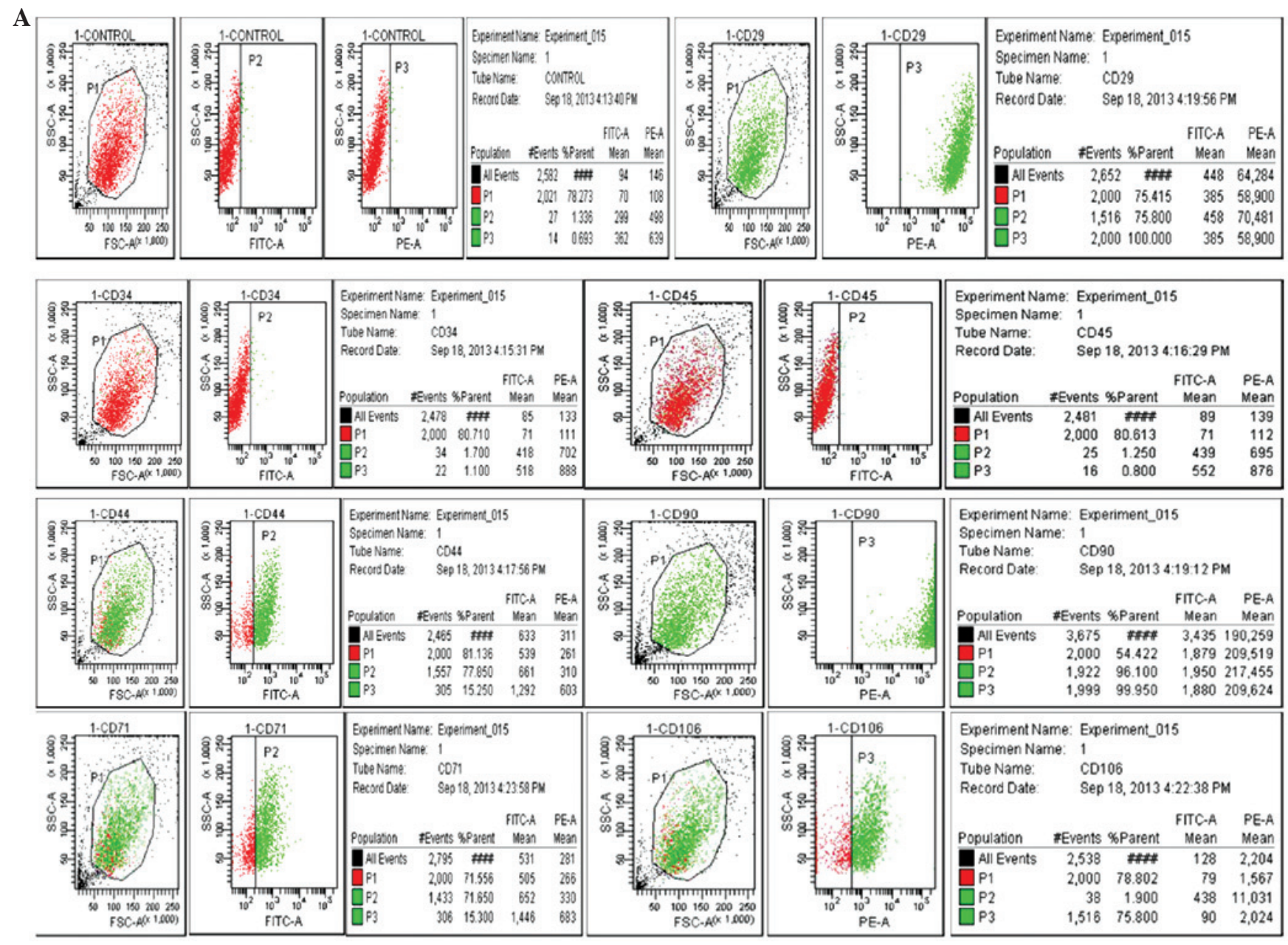

B

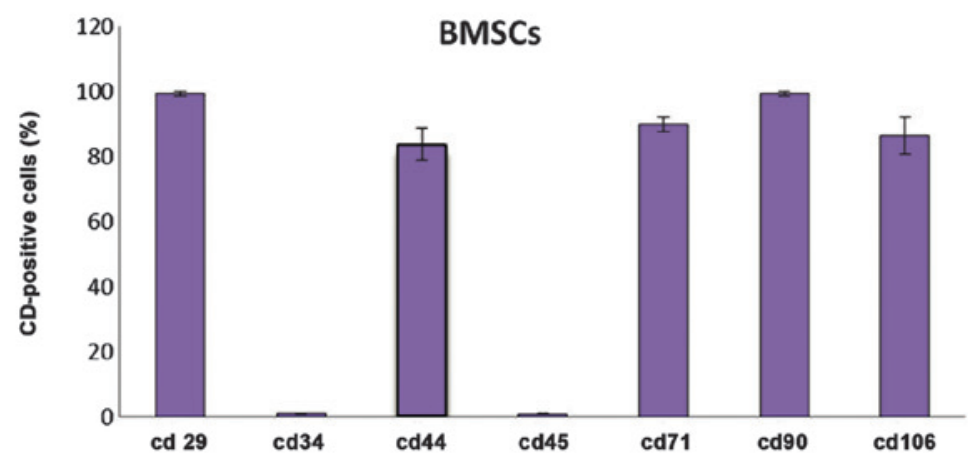

Figure 2. Identification and immunophenotypic characterization of bone marrow mesenchymal stem cells (BMSCs) isolated from Sprague Dawley rats, as determined by flow cytometric analysis of surface antigens. (A) The cells were harvested and stained with fluorescein isothiocyanate-conjugated anti-rat CD29, CD34, CD44, CD45, CD71, CD90, CD106. The BMSCs were negative for CD34, CD45, but positive for mesenchymal markers CD29, CD44, CD71, CD90 and CD106. (B) Statistical analysis of numerous samples. Fourth passage BMSCs CD marker expression was as follows: CD29 (99.25 $\pm 0.82 \%), C D 34$ (1.00 $\pm 0.20 \%)$,

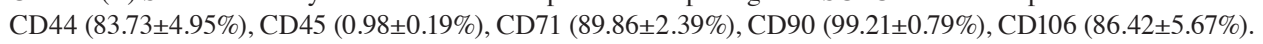

Transduction efficiency of NGF and bFGF lentiviral vectors into BMSCs. A total of $72 \mathrm{~h}$ post-transduction, the transduction efficiency of bFGF and NGF into BMSCs was determined. Western blotting was used to detect the protein expression levels of the two genes in the BMSCs. As shown in Fig. 3, untransfected BMSCs express little NGF and bFGF, thus suggesting that BMSCs rarely secrete the two proteins. Conversely, the two proteins were significantly overexpressed in the BMSCs transfected with the lentiviral vectors. These results indicated that the expression levels of NGF and bFGF may be increased in BMSCs transfected with specific lentiviral vectors.

Differentiation of co-transfected BMSCs. BMSC morphology was observed $72 \mathrm{~h}$ post-transduction. The cells were retracted towards their flat cell nucleus forming a compressed multipolar spherical cell body, producing membranous, process-like extension peripherally. These 

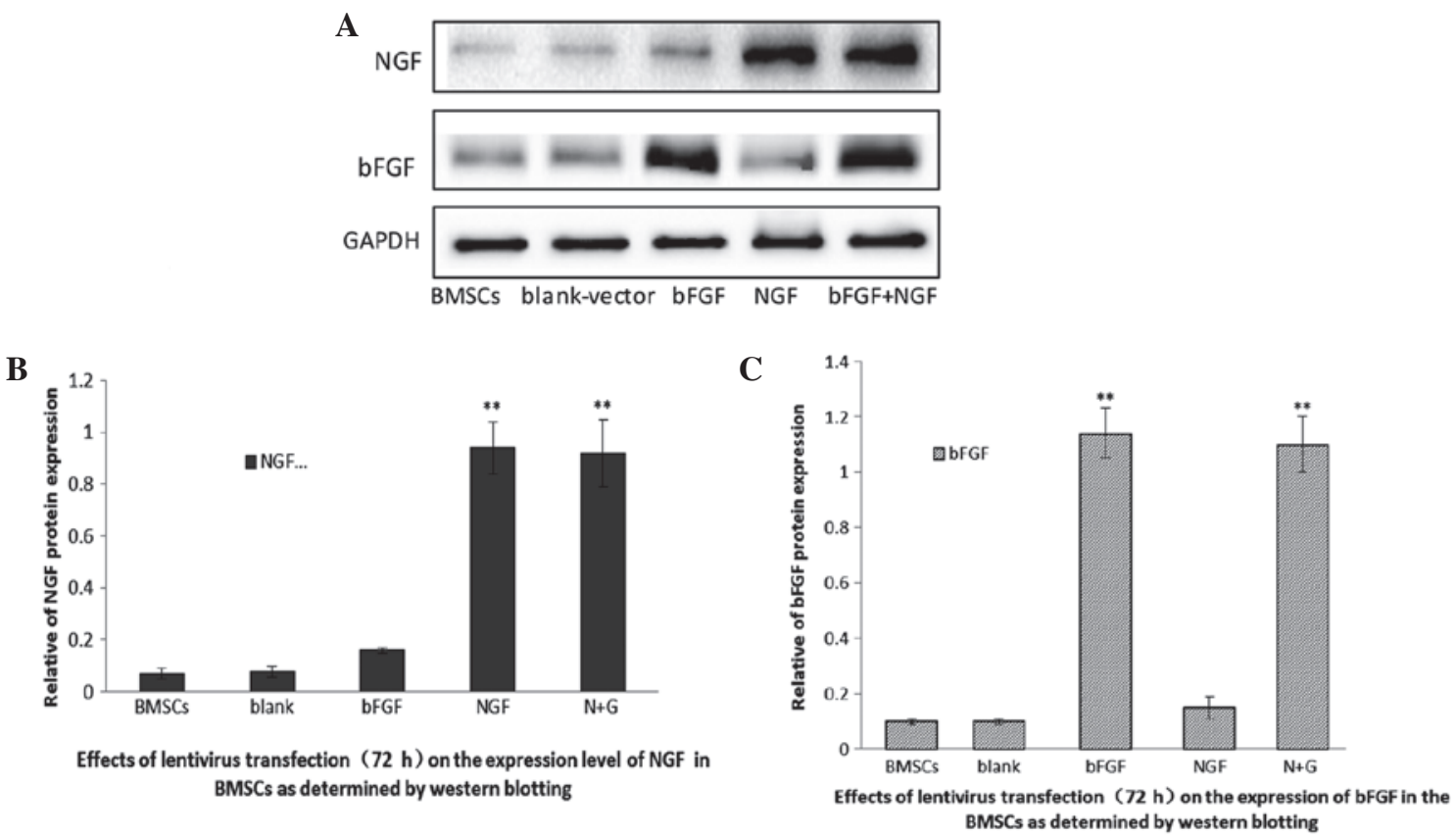

Figure 3. Characterization of nerve growth factor (NGF) and basic fibroblast growth factor (bFGF) lentiviral transfection of bone marrow mesenchymal stem cells (BMSCs). The expression levels of NGF and bFGF proteins in the various transfected cells was determined by western blotting $72 \mathrm{~h}$ post-transfection. (A) The plv-NGF-transfected BMSCs and the plv-NGF-bFGF co-transfected BMSCs expressed NGF protein, whereas the remaining groups secreted little NGF protein. The plv-bFGF-transfected BMSCs and the plv-NGF-bFGF co-transfected BMSCs expressed bFGF protein, whereas the remaining groups secreted little bFGF protein. (B) The data are presented as the mean \pm standard deviation (SD) from five independent groups $(\mathrm{n}=3)$. ${ }^{* *} \mathrm{P}<0.01$, as compared with the untransfected BMSCs, the plv-blank-transfected BMSCs, and the plv-bFGF-transfected BMSCs. (C) The data are presented as the mean \pm SD ( $\mathrm{n}=3$ ). ${ }^{* *} \mathrm{P}<0.01$, as compared with the untransfected BMSCs, the plv-blank-transfected BMSCs, and the plv-NGF-transfected BMSCs.

cells have a significantly higher refractive index difference than the neuronal cell bodies involved in the process, similar to the termination structure of the growth cone. The expansion of putative filopodia demonstrated that the transfected BMSCs have the potential to differentiate into neural-like cells. The number of cells and the neuronal morphology were both increased, and the cell synapse gradually formed over the $72 \mathrm{~h}$. Conversely, the untransfected BMSCs remained spindle-shaped. Subsequently, nestin (a neural stem cell marker), NSE (a neuronal marker), and GFAP (an astrocyte marker) were used to identify differentiated neural cells (Fig. 4A). Green immunofluorescence intensity was used to detect the various neuron markers. NGF and bFGF co-transfected BMSCs exhibited higher intensities of green fluorescence. Western blotting detected the protein expression levels of NSE, nestin and GFAP in the various groups (Fig. 4B). Notably, relative protein expression levels were higher in the bFGF and NGF co-transfected group, as compared with the NGF-transfected group or the bFGF-transfected group (Fig. 4C). Positive staining rates were $8.1 \pm 1.7 \%$, $28.57 \pm 6.29 \%$ and $54.29 \pm 5.71 \%$ for nestin; $11 \pm 2.66 \%$, $40.14 \pm 5.81 \%$ and $69.29 \pm 5.82 \%$ for NSE; and $6.43 \pm 2.15 \%$ and $15.29 \pm 2.81 \%$, for GFAP. In addition, the co-transfected BMSCs and NGF-transfected BMSCs possessed increased numbers of differentiating neuron-like cells, as compared with the bFGF-transfected group (Fig. 4D).

Expression levels of $\beta$-tubulin III in differentiated neuron-like cells at various passages. $\beta$-tubulin III, which is a component of the cytoskeleton, is a neuronal specific tubulin considered to be an indicator of early neuronal development (23-26). Under laser scanning confocal microscopy, early neuronal-like cells were identified in the initial passage of the transfected cells, as they began to differentiate. When the cells had differentiated to the second passage, weak green fluorescence, indicating $\beta$-tubulin III expression, gathered in the perinuclear area and extended to form a network structure. When the cells had differentiated to the fourth passage, the presence of green fluorescent reactants was significantly increased, indicating a higher expression of $\beta$-tubulin III. In addition, strong perinuclear expression of $\beta$-tubulin III was also detected. After 6 passages, the cell structure was clearer, and the fluorescence intensity surrounding the nucleus at the synapse was stronger. At the eighth passage, nuclear green fluorescence was decreased, and extrusion was detected (Fig. 5A). In the various transfected BMSC groups, from passage 2-8, the protein expression of $\beta$-tubulin III was initially increased, and was then decreased. In the control BMSCs and plv-blank-transfected BMSCs the protein expression of $\beta$-tubulin III was absent, whereas in the bFGF-transfected group, the protein expression of $\beta$-tubulin III was detected, but at small levels. The protein expression levels of $\beta$-tubulin III were higher in the fourth and sixth passages, as compared with in the second and fourth passages,respectively, of BMSCs. Although in the NGF- and bFGF- transfected BMSCs, the protein expression levels increased from passage $2-6$, at passage 8 , the protein expression levels were markedly lower. The differences between the two groups were statistically significant (Fig. 5B).

At the second passage, in the NGF and bFGF co-transfected BMSCs group, the reaction rate with $\beta$-tubulin III was $61.40 \pm 1.89 \%$; in the NGF-transfected BMSCs group the reaction rate was $45 \pm 1.2 \%$; and in the bFGF-transfected 
A
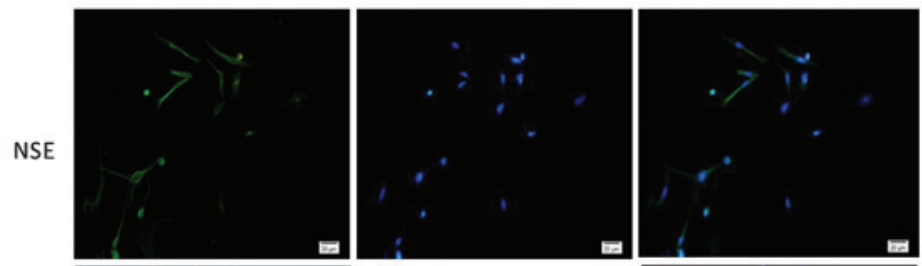

B
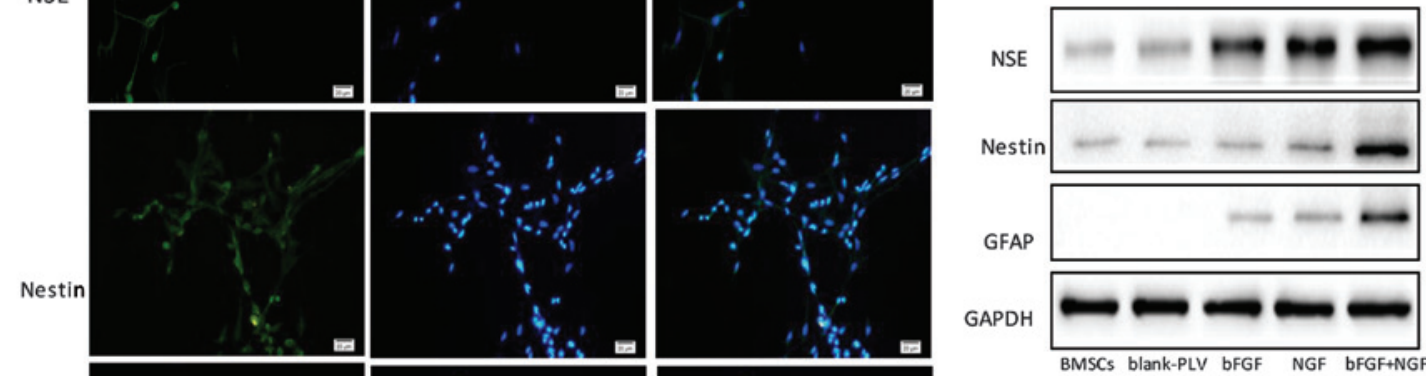

GFAP
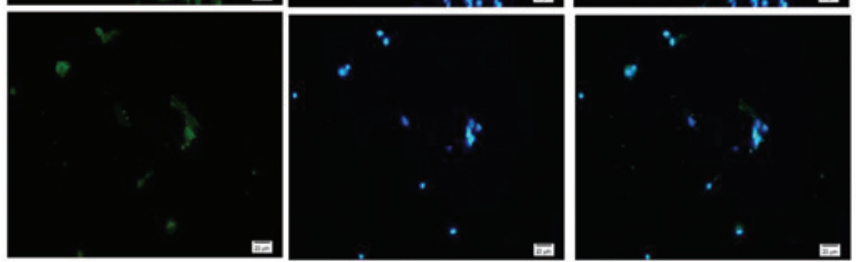

$\mathbf{C}$

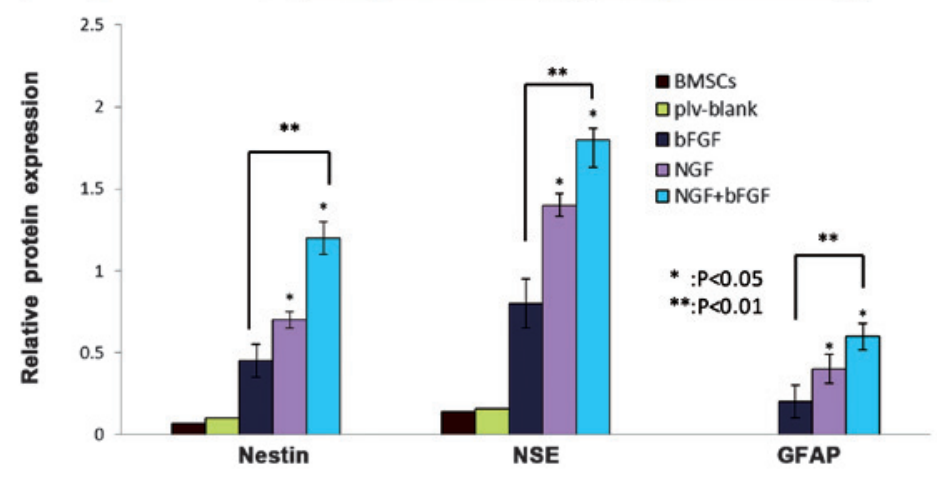

Scale bar $=20 \mu \mathrm{m}$

The neuron marker protein expressed in each differentiated group after transfected at $72 \mathrm{~h}$

D

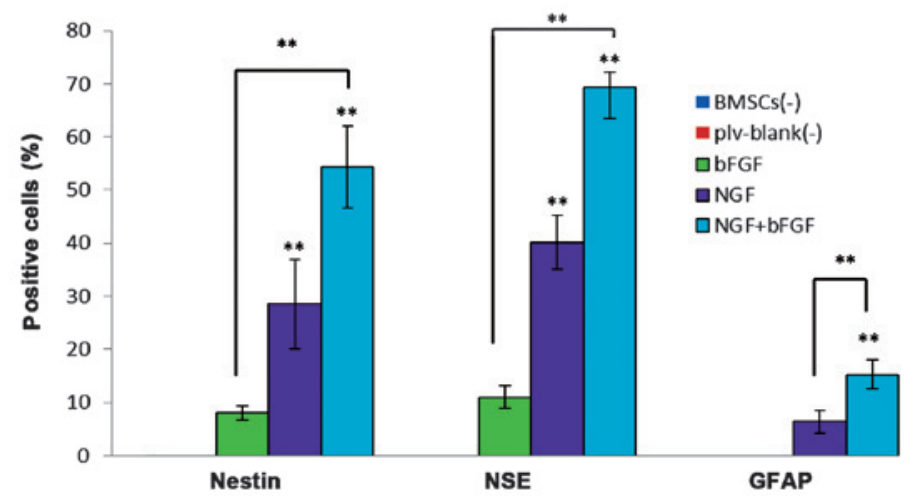

Figure 4. Protein expression levels of nestin, neuron-specific enolase (NSE) and glial fibrillary acidic protein (GFAP) neuron-markers in nerve growth factor (NGF)- and basic fibroblast factor (bFGF)-transfected bone marrow mesenchymal stem cells (BMSCs) $72 \mathrm{~h}$ post-transfection. (A) Differentiated cells were stained with nestin, NSE and GFAP antibodies and counterstained with DAPI (blue). Scale bar=20 $\mu \mathrm{m}$. The NGF and bFGF co-transfected BMSCs exhibited increased protein expression of nestin, NSE and GFAP, as demonstrated by a marked increase in green fluorescence after $72 \mathrm{~h}$, detected by fluorescence microscopy. (B) Western blotting detected the protein expression levels of neuron markers in the transfected BMSCs after $72 \mathrm{~h}$. The expression levels of the neuron markers were higher in the co-transfected BMSCs group, as compared with the other groups. The untransfected BMSCs and plv-blank-transfected cells exhibited a slight expression of neuron markers. (C) Western blot analysis of the protein expression levels of nestin, NSE and GFAP in the untransfected BMSCs group, plv-blank-transfected BMSCs group, bFGF-transfected BMSCs group, NGF-transfected BMSCs group, and NGF and bFGF co-transfected BMSCs group. Data show the protein levels normalized to GAPDH, and are presented as the mean \pm standard deviation (SD) $\left(\mathrm{n}=5\right.$ ). ${ }^{*} \mathrm{P}<0.05$, co-transfected group vs. NGF-transfected group, and NGF-transfected group vs. bFGF-transfected group; ${ }^{* *} \mathrm{P}<0.01$, co-transfected group vs bFGF-transfected group.(D) Percentage of neural cells positive for nestin, NSE and GFAP in the bFGF-transfected BMSCs group, NGF-transfected BMSCs group, and co-transfected BMSCs group. Data are presented as the mean $\pm \mathrm{SD}(\mathrm{n}=5)$. ${ }^{* *} \mathrm{P}<0.01$, each group was significantly different, as compared with the other groups.

BMSCs group the reaction rate was $16.10 \pm 0.5 \%$. At the fourth passage, the reaction rate with $\beta$-tubulin III was $68 \pm 7.20 \%, 50 \pm 2.10 \%$, and $13 \pm 0.50 \%$ in the co-transfected, NGF-transfected, and bFGF-transfected cells, respectively.
At the sixth passage, the reaction rate with $\beta$-tubulin III was $70.40 \pm 9.24 \%, 55 \pm 3.60 \%$ and $17 \pm 3.80 \%$ in the co-transfected, NGF-transfected, and bFGF-transfected cells, respectively. In addition, at the eighth passage, the reaction 
A
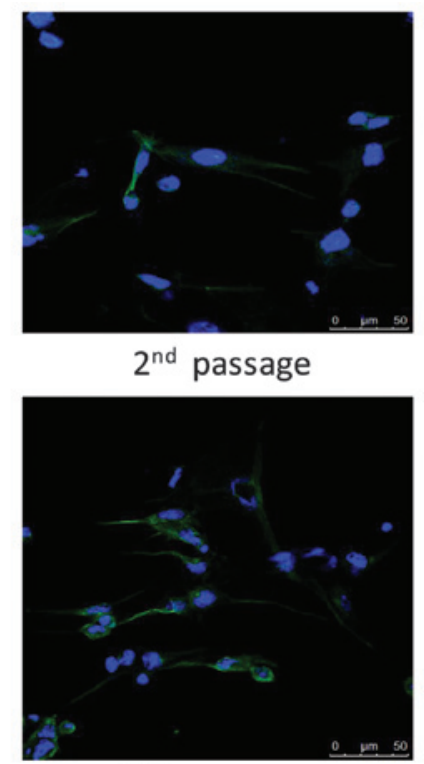

$8^{\text {th }}$ passage

B

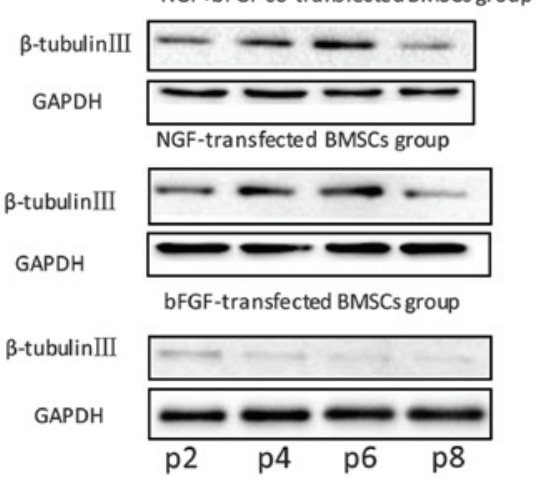

BMSCs group $\beta$-tubulinIII (-)

PLV-blank-transfected in BMSCs group $\beta$-tubulinIII(-)

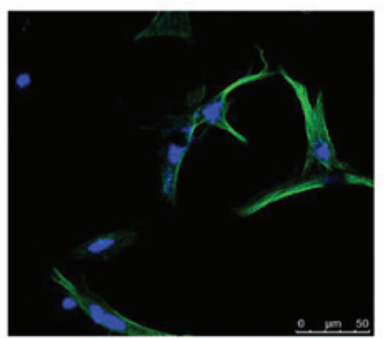

$4^{\text {th }}$ passage

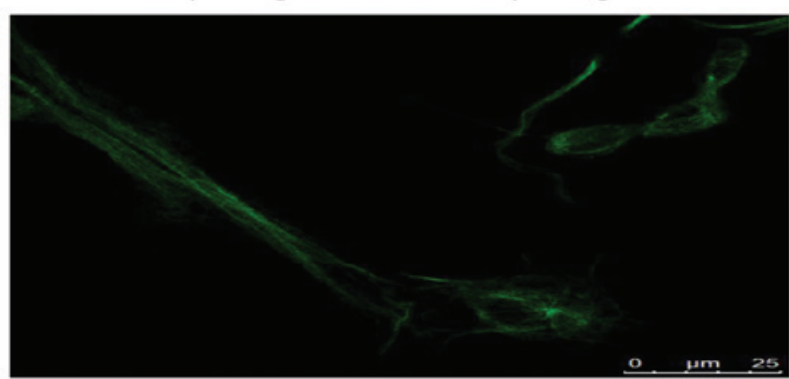

Differentiated nerve fibers

C

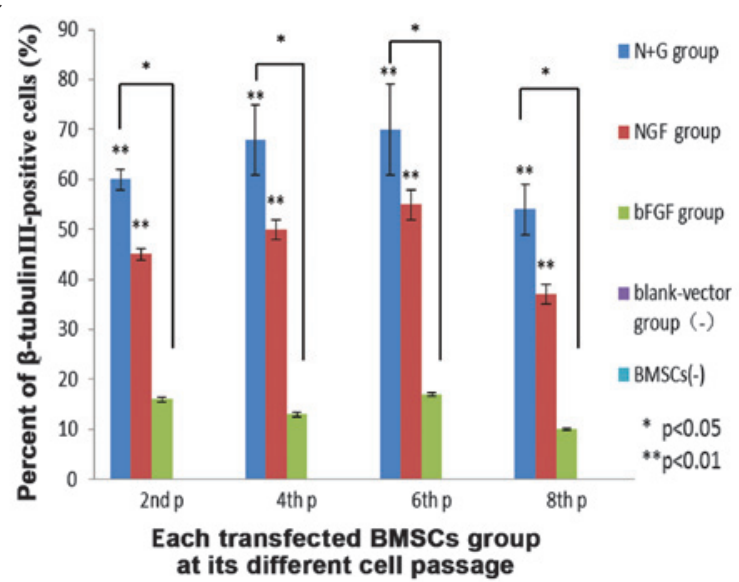

Figure 5. (A) Nerve growth factor (NGF) and basic fibroblast growth factor (bFGF) co-transfected bone marrow mesenchymal stem cells (BMSCs) at passages $2,4,6$, and 8 express $\beta$-tubulin III, as detected by the presence of green fluorescent dye. Scale bar=50 $\mathrm{mm}$. Neuron-like extensions and inter-connections of NGF and bFGF were depicted. Nerve fibers scale bar $=25 \mathrm{~mm}$. All images were obtained with a laser scanning confocal microscope (Zeiss LSM 510). (B) The expression of $\beta$-tubulin III in the various groups of differentiated cells at passages $2,4,6$, and 8 . (C) Flow cytometric analysis of $\beta$-tubulin III expression in the three groups at passages $2,4,6$, and 8 . Data are presented as the mean \pm standard deviation $(n=3)$. ${ }^{*} \mathrm{P}<0.05,{ }^{* * *} \mathrm{P}<0.01$.

rate with $\beta$-tubulin III was $54.57 \pm 5.15 \%, 37.18 \pm 1.27 \%$ and $11.28 \pm 0.26 \%$ in the co-transfected, NGF-transfected, and bFGF-transfected cells, respectively (Fig. 5C). These results suggested that $\beta$-tubulin III expression was highest in the co-transfected BMSCs, as compared with the other groups. Furthermore, co-transfected BMSCs may differentiate into more sophisticated neuron-like cells, as compared with the remaining groups.

AKT and ERK signaling pathways are involved in the neural differentiation of transfected BMSCs. MAPK has a significant role in the growth and differentiation of BMSCs. In the present study, western blotting was used to examine the protein expression levels of ERK in the various groups. As shown in Fig. 6, the expression levels of p-ERK differed between the groups. The expression levels of p-ERK were increased in the NGF-transfected and co-transfected groups, and no significant changes were detected between the expression of p-ERK in the

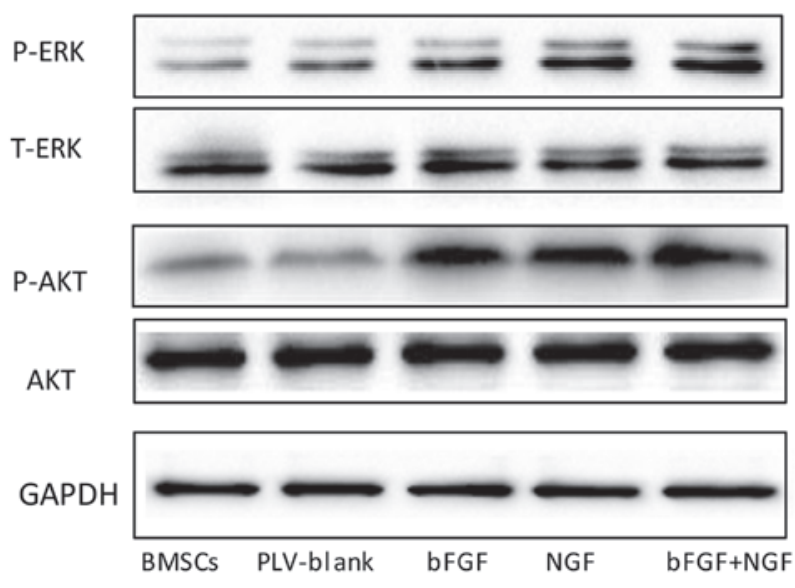

Figure 6. Western blotting was conducted to determine whether extracellular signal-regulated kinases (ERK) and AKT pathways were associated with the differentiation of bone marrow mesenchymal stem cells (BMSCs). The expression of endogenous phosphorylated (p)-AKT and p-ERK was also detected in BMSCs. T, total; bFGF, basic fibroblast growth factor; NGF, nerver growth factor. 
BMSCs and plv-blank-transfected BMSCs. These results indicated that the MAPK pathway may manipulate the neuronal cell differentiation of BMSCs. Transduction of BMSCs with bFGF and NGF enhanced the phosphorylation of ERK (Fig. 6). In addition, the western blot analysis demonstrated that NGFand bFGF-transfected BMSCs exhibited increased expression levels of AKT phosphorylation. However, no significant differences in the expression levels of AKT were detected between the control BMSCs and blank-vector-transfected BMSCs. These results suggested that AKT may also be involved in BMSCs neural differentiation.

\section{Discussion}

BMSCs have the potential of multi-directional differentiation (22). BMSCs have been reported to exert beneficial effects for use in clinical treatment and cell regeneration, and may be regarded as an advanced and promising therapeutic tool $(23,27,28)$. Neurotrophins have a predominant role in development and differentiation, and stimulate the tightly regulated process of BMSC neural differentiation. In order to take advantage of BMSCs and neurotrophins for nerve cell regeneration, it is necessary to research the specific mechanisms underlying neural differentiation. Delcroix et al (27) demonstrated that pre-treatment with bFGF was able to enhance neural specification, and Fan et al (28) reported that NGF and vascular endothelial growth factor enhance angiogenic effects in vivo.

It is convenient to use BMSCs for the treatment of injured tissue. Conversely, neurotrophic factors secreted by transfected BMSCs are beneficial for the restoration of injured tissue. BMSCs are able to promote survival of grafted cells, and also secrete a sufficient amount of mature neurotrophic factors.

bFGF is expressed in the embryonic and adult central and peripheral nervous systems, and maintains the survival of neuronal and glial cells, promotes sympathetic and parasympathetic nervous axon growth, and promotes the repair of damaged nerves and neurite outgrowth (19). In addition, bFGF is able to induce differentiation of adrenal pheochromocytoma cells-12 (29), and extend human neural progenitor cell nerve regeneration and neural differentiation (7). Therefore, bFGF is considered an important growth factor. NGF is a significant neuropeptide signal, which can induce BMSC neural differentiation, and regulate cell proliferation and cell survival growth (7). Lentiviral vectors may be used to stably transfect cells, and genes integrated into the genome of target cells using lentiviral vectors exhibit long-term expression and immune response.

The present study constructed NGF and bFGF co-transfected SD rat BMSCs, in order to determine the effects of overexpression of these two genes on differentiation into neuron-like cells. The results of the present study demonstrated that NGF and bFGF co-transfection was able to induce the differentiation of BMSCs into neuron-like cells; the co-transfected group had the highest proportion of neuron-like cell differentiation, as compared with the other groups.

In the present study, BMSCs of stable growth were selected as cells to be transfected. Once the NGF and bFGF genes were successfully recombined with the lentiviral vector in vitro, western blotting was used to detect the expression levels of each of the proteins. In addition to spindle-shaped and polygonal cell morphology, a series of positive $\mathrm{CD}$-antigenic markers was used for identification of successfully transfected BMSCs through flow cytometry. In the control BMSCs and blank vector-transfected cells a low expression of NGF was detected. This finding indicated that, the lentivirus-vector transfection and bFGF may enhance the BMSCs, while BMSCs can themselves secrete a small quantity of NGF. These results indicated that transfection with the two genes effectively increased the bFGF and NGF protein expression levels in BMSCs.

The results of the present study also demonstrated that the NGF and bFGF co-transfected cells exhibited much higher neuron-marker expression, as compared with the other groups. In the fourth and sixth passages, $\beta$-tubulin III protein expression was detected, as mature neuronal microtubule structures were formed and migration increased, due to the maturation of the cytoskeleton. Intracellular microtubules of $\beta$-tubulin III consist of a long line of aligned fibrils formed by a hollow tubular structure. The results of the present study indicated that transfected BMSCs were able to differentiate from the polar cells of spindle-shaped and polygonal morphology into neuron-like cells. This process occurred alongside increasing $\beta$-tubulin III expression.

$\beta$-tubulin III, which is a neuron-specific tubulin, is a component of the cytoskeleton, and has been considered a sign of early neuronal development. $\beta$-tubulin III has an important role in the development of the neuron cytoskeleton during neural differentiation and maturation $(26,30)$. In the present study, in the second passage BMSCs, $\beta$-tubulin III expression was weak, and was mainly distributed in the nucleus, during the continual development of neurons. When the cells reached passages 4 and 6 , $\beta$-tubulin III expression increased and extended outwards. It is possible that the synthesized intracellular rough endoplasmic reticulum gradually begins to participate in the formation of microtubules, thus extending the projections of the nucleus. However, as the neuron cell matured to the eighth generation, $\beta$-tubulin III expression was reduced, and extension out of the cells was decreased. Western blotting also demonstrated that in early neural differentiation, $\beta$-tubulin III expression gradually increased, and then decreased at the eighth passage. Due to the intracellular distribution and content of $\beta$-tubulin III, it is believed that $\beta$-tubulin III has an important role in the neural differentiation of axonal growth. These results suggest that neuronal development may be associated with microtubule polymerization. In the early stages of neural differentiation, it may be hypothesized that $\beta$-tubulin III promotes the formation of microtubules, thereby affecting neural differentiation and neuron outgrowth. In addition, NGF and bFGF may enhance the expression of $\beta$-tubulin III.

The results of the present study provide compelling evidence that NGF and bFGF have a critical role in the high-efficiency differentiation of BMSCs into neuron-like cells. It has been previously reported exogenous factor-induced differentiation of MSCs, suggesting that the ERK pathway is involved in the neural differentiation of BMSCs $(27,31)$. The present study demonstrates that NGF and BFGF co-transfected BMSCs exhibited an increased expression of ERK phosphorylation in BMSCs, as well as increased proliferation and differentiation. These results indicated that MAPKs may regulate BMSCs, thus enhancing phosphorylation in NGF and bFGF-transfected 
cells. Concurrently, the AKT pathway may also be involved, which is able to inhibit cell apoptosis. The results of the present study demonstrated that NGF- and bFGF-transfected BMSCs exhibited increased expression levels of p-AKT, whereas the control BMSCs and blank-vector-transfected BMSCs exhibited lower expression of AKT phosphorylation, thus suggesting that that AKT pathway is involved in regulation of BMSC neural differentiation.

In conclusion, bFGF and NGF co-transfected BMSCs exhibited enhanced neural specification and response to neuronal commitment of stem cells, resulting in the induction of differentiation and maturation of neuron cells. Furthermore, ERK and AKT signaling pathways were shown to be involved in the neural differentiation of NGF and bFGF co-transfected BMSCs. The present study may influence the use of cell therapy to treat adult nervous system diseases in the future.

\section{Acknowledgements}

The authors of the present study would like to thank Quentin Liu Lab at the Dalian Medical University for their critical demands and technical support. The present study was supported by the National Natural Science Foundation of China (grant no. 81270052).

\section{References}

1. Turgeman G, Pittman DD, Müller R, Kurkali BG, Zhou S, Pelled G, Peyser A, Zilberman Y, Moutsatsos IK and Gazit D: Engineered human mesenchymal stem cells: A novel platform for skeletal cell mediated gene therapy. J Gene Med 3: 240-251, 2001

2. Mohammadi R, Azizi S, Delirezh N, Hobbenaghi R, Amini K and Malekkhetabi P: The use of undifferentiated bone marrow stromal cells for sciatic nerve regeneration in rats. Int J Oral Maxillofac Surg 41: 650-656, 2012.

3. Pereira Lopes FR, Camargo de Moura Campos L, Dias Corrêa J Jr, Balduino A, Lora S, Langone F, Borojevic R and Blanco Martinez AM: Bone marrow stromal cells and resorbable collagen guidance tubes enhance sciatic nerve regeneration in mice. Exp Neurol 198: 457-468, 2006.

4. Wu J, Yu W, Chen Y, Su Y, Ding Z, Ren H, Jiang Y and Wang J: Intrastriatal transplantation of GDNF-engineered BMSCs and its neuroprotection in lactacystin-induced Parkinsonian rat model. Neurochem Res 35: 495-502, 2010.

5. Wen SR, Qi HP, Ren YJ, Liu GJ, Gong FC, Zhong H and Bi S: Expression of $\delta \mathrm{Np} 73$ in hippocampus of APP/PS1 transgenic mice following GFP-BMSCs transplantation. Neurol Res 33: $1109-1114,2011$.

6. Ding J, Cheng Y, Gao S and Chen J: Effects of nerve growth factor and Noggin-modified bone marrow stromal cells on stroke in rats. J Neurosci Res 89: 222-230, 2011.

7. Zhu H1 Yang A, Du J, Li D, Liu M, Ding F, Gu X and Liu Y: Basic fibroblast growth factor is a key factor that induces bone marrow mesenchymal stem cells towards cells with Schwann cell phenotype. Neurosci Lett 559: 82-87, 2014.

8. Mohammadi R, Azizi S, Delirezh N, Hobbenaghi R and Amini K: Comparison of beneficial effects of undifferentiated cultured bone marrow stromal cells and omental adipose-derived nucleated cel fractions on sciatic nerve regeneration. Muscle Nerve 43: 157-163, 2011.

9. Wang S, Yaszemski MJ, Knight AM, Gruetzmacher JA, Windebank AJ and Lu L: Photo-crosslinked poly(epsilon-caprolactone fumarate) networks for guided peripheral nerve regeneration: Material properties and preliminary biological evaluations. Acta Biomater 5: 1531-1542, 2009.

10. Crouzier T, McClendon T, Tosun $Z$ and McFetridge PS: Inverted human umbilical arteries with tunable wall thicknesses for nerve regeneration. J Biomed Mater Res A 89: 818-828, 2009.
11. Cuevas P, Carceller F, Garcia-Gómez I, Yan M and Dujovny M: Bone marrow stromal cell implantation for peripheral nerve repair. Neurol Res 26: 230-232, 2004.

12. Liu WG, Wang ZY and Huang ZS: Bone marrow-derived mesenchymal stem cells expressing the bFGF transgene promote axon regeneration and functional recovery after spinal cord injury in rats. Neurol Res 33: 686-693, 2011.

13. Colafreancesco V and Villoslada P: Targeting NGF pathway for developing neuroprotective therapies for multiple sclerosis and other neurological diseases. Arch Ital Biol 149: 183-192, 2011.

14. Kopen GC, Prockop DJ and Phinney DG: Marrow stromal cells migrate throughout forebrain and cerebellum, and they differentiate into astrocytes after injection into neonatal mouse brains. Proc Natl Acad Sci USA 96: 10711-10716, 1999.

15. Li Y, Chen J and Chopp M: Adult bone marrow transplantation after stroke in adult rats. Cell Transplant 10: 31-40, 2001.

16. Li Y, Chopp M, Chen J, Wang L, Gautam SC, XU YX and Zhang Z: Intrastriatal transplantation of bone marrow nonhematopoietic cells improves functional recovery after stroke in adult mice. J Cereb Blood Flow Metab 20: 1311-1319, 2000.

17. Chopp M, Zhang XH, Li Y, Wang L, Chen J, Lu D, Lu M and Rosenblum M: Spinal cord injury in rat: Treatment with bone marrow stromal cell transplantation. Neuroreport 11: 3001-3005, 2000.

18. Maden M: Retinoic acid in the development, regeneration and maintenance of the nervous system. Nat Rev Neurosci 8: 755-765, 2007.

19. Bithell A, Finch SE, Hornby MF and Williams BP: Fibroblast growth factor 2 maintains the neurogenic capacity of embryonic neural progenitor cells in vitro but changes their neuronal subtype specification. Stem Cells 26: 1565-1574, 2008.

20. Sanchez-Ramos J, Song S, Cardozo-Pelaez F, Hazzi C, Stedeford T, Willing A, Freeman TB, Saporta S, Janssen W, Patel N, et al: Adult bone marrow stromal cells differentiate into neural cells in vitro. Exp Neurol 164: 247-256, 2000.

21. Mudò G, Bonomo A, Di Liberto V, Frinchi M, Fuxe K and Belluardo N: The FGF-2/FGFRs neurotrophic system promotes neurogenesis in the adult brain. J Neural Transm 116: 995-1005, 2009.

22. Zhu H, Guo ZK, Jiang XX, Li H, Wang XY, Yao HY, Zhang Y and Mao N: A protocol for isolation and culture of mesenchymal stem cells from mouse compact bone. Nat Protoc 5: 550-560, 2010.

23. Katsetos CD, Legido A, Perentes E and Mörk SJ: Class III beta-tubulin isotype: A key cytoskeletal protein at the crossroads of developmental neurobiology and tumor neuropathology. J Child Neurol 18: 851-867, 2003.

24. Wang Z, Deng Q, Zhang X and Zhang J: Treatment of injured neurons with bone marrow stem cells cotransfected by hTERT and Ad-BDNF in vitro. J Mol Neurosci 38: 265-272, 2009.

25. Fan BS and Lou JY: Enhancement of angiogenic effect of co-transfection human NGF and VEGF genes in rat bone marrow mesenchymal stem cells. Gene 485: 167-171, 2011.

26. Tao YX, Xu HW, Zheng Q Y and FitzGibbon T: Noggin induces human bone marrow-derived mesenchymal stem cells to differentiate into neural and photoreceptor cells. Indian J Exp Biol 48: 444-452, 2010.

27. Delcroix GJ, Curtis KM, Schiller PC and Montero-Menei CN: EGF and bFGF pre-treatment enhances neural specification and the response to neuronal commitment of MIAMI cells. Differentiation 80: 213-227, 2010.

28. Fan BS and Lou JY: Enhancement of angiogenic effect of co-transfection human NGF and VEGF genes in rat bone marrow mesenchymal stem cells. Gene 485: 167-171, 2011.

29. Tobin JE, Xie M, Le TQ, Song SK and Armstrong RC: Reduced axonopathy and enhanced remyelination after chronic demyelination in fibroblast growth factor 2 (Fgf2)-null mice: Differential detection with diffusion tensor imaging. J Neuropathol Exp Neurol 70: 157-165, 2011.

30. Fanarraga ML, Avila J and Zabala JC: Expression of unphosphorylated class III beta-tubulin isotype in neuroepithelial cells demonstrates neuroblast commitment and differentiation. Eur J Neurosci 11: 516-527, 1999.

31. Lam HJ, Patel S, Wang A, Chu J and Li S: In vitro regulation of neural differentiation and axon growth by growth factors and bioactive nanofibers. Tissue Eng Part A 16: 2641-2648, 2010. 